YITP-05-76

December 2005

\title{
Constraining Dark Matter in the MSSM at the LHC
}

\author{
M.M. Nojiri \\ YITP, Kyoto University, Kyoto 606-8502, Japan. \\ G. Polesello \\ INFN, Sezione di Pavia, Via Bassi 6, I-27100 Pavia, Italy. \\ D.R. Tovey \\ Department of Physics and Astronomy, University of Sheffield, \\ Hounsfield Road, Sheffield S3 $7 R H, U K$.
}

\begin{abstract}
In the event that R-Parity conserving supersymmetry (SUSY) is discovered at the LHC, a key issue which will need to be addressed will be the consistency of that signal with astrophysical and non-accelerator constraints on SUSY Dark Matter. This issue is studied for the SPA benchmark model based on measurements of end-points and thresholds in the invariant mass spectra of various combinations of leptons and jets. These measurements are used to constrain the soft SUSY breaking parameters at the electroweak scale in a general MSSM model. Based on these constraints, we assess the accuracy with which the Dark Matter relic density can be measured.
\end{abstract}

PACS: 12.60.Jv; 04.65.+e; 95.35.+d

Keywords: LHC physics; supersymmetry; SUGRA; dark matter; relic density 


\section{Introduction}

The complementarity of LHC SUSY measurements and direct and indirect searches for neutralino Dark Matter is an important topic to study given the increasingly strong astrophysical evidence for cold Dark Matter in the universe [1, 2, 3]. Assuming that R-Parity conserving SUSY is discovered at the LHC, an interesting question will arise regarding the compatibility of that signal with existing relic density constraints (e.g. $0.094<\Omega_{\chi} h^{2}<0.129$ at $2 \sigma$ from WMAP data [1, 2, 3]), and the implications it has for terrestrial Dark Matter searches.

In a previous paper [4 we addressed these issues within the context of the minimal supergravity (mSUGRA) class of SUSY models incorporating gravity-mediated SUSY breaking [5]. There is a great advantage in studying models such as the mSUGRA ones, where a definite pattern of SUSY breaking is imposed at the unification scale. These models are in fact described with only a small number of independent parameters. It is therefore possible, through a limited number of measurements to fully constrain the model, and extract very precise predictions for physical quantities related to Dark Matter. However, the mSUGRA model is strongly constrained by the WMAP data, and we do not know whether from the LHC measurements an unambiguous determination of the high scale behaviour of the SUSY theory will be possible. It is therefore interesting to study a generic model in which the weak-scale SUSY breaking parameters are independent, such as the Minimal Supersymmetric Standard Model (MSSM), and verify to what level the LHC measurements can constrain the neutralino relic density and the cross-sections for direct Dark Matter detection.

Some recent papers [6] 7], in the framework of International Linear Collider (ILC) studies, based on generic and sometimes restrictive assumptions on the measurement potential of the LHC, conclude for a very limited potential of the LHC experiments in constraining the neutralino relic density in a generic MSSM. The aim of this paper is to investigate in detail, by carefully taking into account published studies, what are the effective limitations of the LHC measurements in this field for a specific representative model for which sufficient information is available.

We choose to study one particular point of the MSSM parameter space, which was adopted as a benchmark point by the SPA group [8]. This model is defined in terms of the parameters of the mSUGRA model $\left(m_{0}=70 \mathrm{GeV}, m_{1 / 2}=250 \mathrm{GeV}, A_{0}=-300 \mathrm{GeV}, \tan \beta=10, \mu>0\right)$. This is a modification of the point SPS1a, essentially achieved by lowering $m_{0}$ from 100 to $70 \mathrm{GeV}$, originally defined in Ref. 9, to take into account the WMAP results. It is also very near to Point $\mathrm{B}^{\prime}$ of $\left[10\right.$. This model lies in the 'bulk' region of the $m_{0}-m_{1 / 2}$ mSUGRA plane where the relic density is reduced to a small value by $\tilde{\chi}_{1}^{0}$ annihilation to leptons or neutrinos via $t$-channel slepton, stau and sneutrino exchange. The values of the MSSM soft parameters and of the sparticle masses were computed with the program ISASUSY 7.71 [1], taking the tree-level values for the sparticle masses, and are given in Table 11. The Lightest Supersymmetric Particle (LSP) relic density was computed based on these inputs using the program micrOMEGAs 1.3.6 [12], and the resulting value is $\Omega_{\chi} h^{2}=0.108$, well within the WMAP range. The main annihilation processes contributing to the calculation of the relic density are given in Table 2] As discussed above we take this point as a benchmark, but we analyze it without assuming a predefined relationship among the SUSY breaking parameters.

In Section 2 we describe the LHC measurements which can be used to constrain SUSY at the considered model point. In Section 3 we develop a strategy to constrain the MSSM parameters relevant to the calculation of the neutralino relic density. In Section 4 we discuss 
the use of these constraints to calculate the $\tilde{\chi}_{1}^{0}$ relic density. Finally in Section 5 we review the results and discuss the general applicability of the technique.

\begin{tabular}{|c|c|c|c|}
\hline Sparticle & mass $(\mathrm{GeV})$ & Sparticle & mass $(\mathrm{GeV})$ \\
\hline \hline$\tilde{\chi}_{1}^{0}$ & 97.2 & $\tilde{\chi}_{2}^{0}$ & 180.1 \\
$\tilde{\chi}_{3}^{0}$ & 398.4 & $\tilde{\chi}_{4}^{0}$ & 413.8 \\
$\tilde{\ell}_{L}$ & 189.4 & $\tilde{\ell}_{R}$ & 124.1 \\
$\tilde{\tau}_{1}$ & 107.7 & $\tilde{\tau}_{2}$ & 194.2 \\
$\tilde{t}_{1}$ & 347.3 & $\tilde{t}_{2}$ & 562.3 \\
$\tilde{u}_{L}$ & 533.3 & $\tilde{g}$ & 607.0 \\
$h$ & 116.8 & $A$ & 424.6 \\
\hline
\end{tabular}

Table 1: Masses of the sparticles in the considered model as calculated at tree level with ISAJET 7.71 [11]

\begin{tabular}{|l|r|}
\hline Process & Fraction \\
\hline \hline$\tilde{\chi}_{1}^{0} \tilde{\chi}_{1}^{0} \rightarrow \ell^{+} \ell^{-}$ & $40 \%$ \\
$\tilde{\chi}_{1}^{0} \tilde{\chi}_{1}^{0} \rightarrow \tau^{+} \tau^{-}$ & $28 \%$ \\
$\tilde{\chi}_{1}^{0} \tilde{\chi}_{1}^{0} \rightarrow \nu \bar{\nu}$ & $3 \%$ \\
$\tilde{\chi}_{1}^{0} \tilde{\tau}_{1} \rightarrow Z \tau$ & $4 \%$ \\
$\tilde{\chi}_{1}^{0} \tilde{\tau}_{1} \rightarrow A \tau$ & $18 \%$ \\
$\tilde{\tau}_{1} \tilde{\tau}_{1} \rightarrow \tau \tau$ & $2 \%$ \\
\hline
\end{tabular}

Table 2: Fractional contribution of different annihilation processes to the LSP relic density in the considered model. The relic density was calculated with the program micrOMEGAs 1.3.6 [12].

\section{The measurement of SUSY parameters at the LHC}

The development of techniques for measuring parameters characterizing SUSY models has been the subject of much investigation in the last few years, as documented in Ref. 13, 14, 15, and is still a very active field of investigation.

The basic issue is that the presence of two invisible particles in the final state renders the direct measurement of sparticle masses through the detection of invariant mass peaks impossible. Alternative techniques have therefore been developed, based on the exclusive identification of long cascades of two body-decays. It was demonstrated [13, 14] that if a sufficiently long chain can be identified (at least three successive two-body decays), the thresholds and endpoints of the various possible invariant mass combinations among the identified products can be used to achieve a model-independent measurement of the masses of the involved sparticles. Once the masses of the lighter sparticles are obtained with this procedure, in particular the mass of the LSP, additional sparticle masses can be measured through the identification of other shorter decay chains. This program has been carried out recently for the SPS1a model 
point 16, assuming the performance of the ATLAS detector. This study results in a number of measurements of observables which are related to the masses of the sparticles by known algebraic relations. For the work presented here, we assume for the corresponding observables in the SPA model the same errors as the ones obtained in the full analysis for Point SPS1a. The position of the kinematic edges is quite similar in the two points, due to the fact that only the slepton spectrum is modified by the $30 \%$ change in $m_{0}$, and the total production cross-section is somewhat higher in the new point, which should result is somewhat smaller statistical errors. We further checked that the mass spectrum for the SPA model does not present degeneracies in the sparticle masses which would severely reduce the transverse momenta of the visible decay products and thence the experimental selection efficiency. The considered measurements are give in Table 3. The meaning of the different observables, and their expression in terms of sparticle masses is given in Ref. [15]. The scale error is derived from the assumption of a control on the lepton energy scale at the level of $0.1 \%$, and of the jet energy scale at the level of $1 \%$ [14]. Following [17, the $1 \%$ jet scale uncertainty reflects in $\mathrm{a} \sim 0.5 \%$ uncertainty on the position of the edges involving jets and leptons.

\begin{tabular}{|l|c|c|c|c|}
\hline & & \multicolumn{3}{|c|}{ Errors } \\
Variable & Value $(\mathrm{GeV})$ & Stat+Sys $(\mathrm{GeV})$ & Scale $(\mathrm{GeV})$ & Total \\
\hline \hline$m_{\ell \ell}^{\text {max }}$ & 81.2 & 0.03 & 0.08 & 0.09 \\
$m_{\ell \ell q}^{\text {max }}$ & 425.3 & 1.4 & 2.1 & 2.5 \\
$m_{\ell q}^{\text {low }}$ & 266.9 & 0.9 & 1.3 & 1.6 \\
$m_{\ell q}^{\text {high }}$ & 365.9 & 1.0 & 1.8 & 2.1 \\
$m_{\ell \ell q}^{\min }$ & 207.0 & 1.6 & 1.0 & 1.9 \\
$m_{\left(\ell_{L}\right.}-m\left(\tilde{\chi}_{1}^{0}\right)$ & 92.3 & 1.6 & 0.1 & 1.6 \\
$m_{\ell \ell}^{\max }\left(\tilde{\chi}_{4}^{0}\right)$ & 315.8 & 2.3 & 0.3 & 2.3 \\
$m_{\tau \tau}^{\max }$ & 62.2 & 5.0 & 0.3 & 5.0 \\
\hline
\end{tabular}

Table 3: Summary table of the SUSY measurements which can be performed at the LHC with the ATLAS detector [16] used in this analysis. The central values are calculated with ISASUSY 7.71, using the tree-level values for the sparticle masses. The statistical errors are given for the integrated luminosity of $300 \mathrm{fb}^{-1}$. The uncertainty in the energy scale is taken to result in an error of $0.5 \%$ for measurements including jets, and of $0.1 \%$ for purely leptonic mesurements.

Some additional measurements are considered in this analysis:

- The mass of the light Higgs boson from the decay $h \rightarrow \gamma \gamma$ which for $100 \mathrm{fb}^{-1}$, and for $m_{h}=114 \mathrm{GeV}$, has a statistical uncertainty of $\sim 0.5 \mathrm{GeV}[18$.

- The ratio of branching ratios:

$$
B R\left(\tilde{\chi}_{2}^{0} \rightarrow \tilde{\ell}_{R} \ell\right) / B R\left(\tilde{\chi}_{2}^{0} \rightarrow \tilde{\tau}_{1} \tau\right)
$$

No detailed experimental study is available on this measurement. From a particle-level evaluation of the number of events involved, the error on this quantity will be dominated by the systematic uncertainty in the experimental efficiency for the $\tilde{\tau}_{1}$ channel, in 
particular near threshold where the contribution to the visible ditau mass distribution is significant. We assume here a conservative error of $10 \%$.

- The constraints from searches for heavy Higgs bosons of MSSM, which will be discussed in detail in the following.

Two different types of uncertainties are quoted in Table 3 the combined statistical and systematic uncertainties estimated for each measurement, and general errors on the scales of lepton and hadron energy measurement, which affect all the measured quantities in the same way. Since in many cases the scale uncertainties are dominant it is necessary to take into account the correlations between the different measurements when extracting the constraints on the model parameters. In order to take into account the correlations we use a Monte Carlo

technique relying on the generation of simulated experiments sampling the probability density functions of the measured observables. In frequentist statistics, confidence bands describe the probability that an experiment in a set of identical experiments yields a given value for the measured quantities.

We proceed in the following way:

1. An 'experiment' is defined as a set of measurements, each of which is generated by picking a value from a gaussian distribution with mean given by the central value given in Table 3. The correlation due to energy scale is taken into account by using a second gaussian distribution for the energy scale, and using the same random number for all the measurements sharing the same scale.

2. For each experiment, we extract the constraints on the MSSM model as we will describe in the following.

We obtain as a result of this calculation a set of MSSM models, each of which is the "best" estimate for a given Monte Carlo experiment of the model generating the observed measurement pattern. For each of these models the properties of the LSP Dark Matter candidate and other SUSY particles involved in Dark Matter annihilation can then be calculated, with the spread of obtained results being interpreted as the level of precision with which these properties can be measured by the LHC. As we are working in a general MSSM, some of the parameters will only be loosely constrained, if at all, by the measurements. The spread obtained from the variation of the unconstrained parameters needs to be studied in detail to assess the final prediction precision on DM characteristic.

\section{Extraction of MSSM parameters}

In order to extract the parameters of the MSSM we proceed in a stepwise fashion. We first extract the measurement of the sparticle masses from the measured edges. We do not address here the issue of possible ambiguities which can arise on the identity of the particles involved in the decay chains as discussed in [19] and 20]. The procedure yields an error of $\sim 9 \mathrm{GeV}$ for the masses of the sparticles relevant to this study. We show in the left side of Figure 1 the distribution of the measured $\tilde{\chi}_{1}^{0}$ masses for a set of Monte Carlo experiments. The error on the mass is basically independent on the mass of the sparticle because the errors on masses are strongly correlated. This is because of the algebraical structure of the formulas in [15], [17, which causes the absolute scale to be much more loosely constrained than the difference 

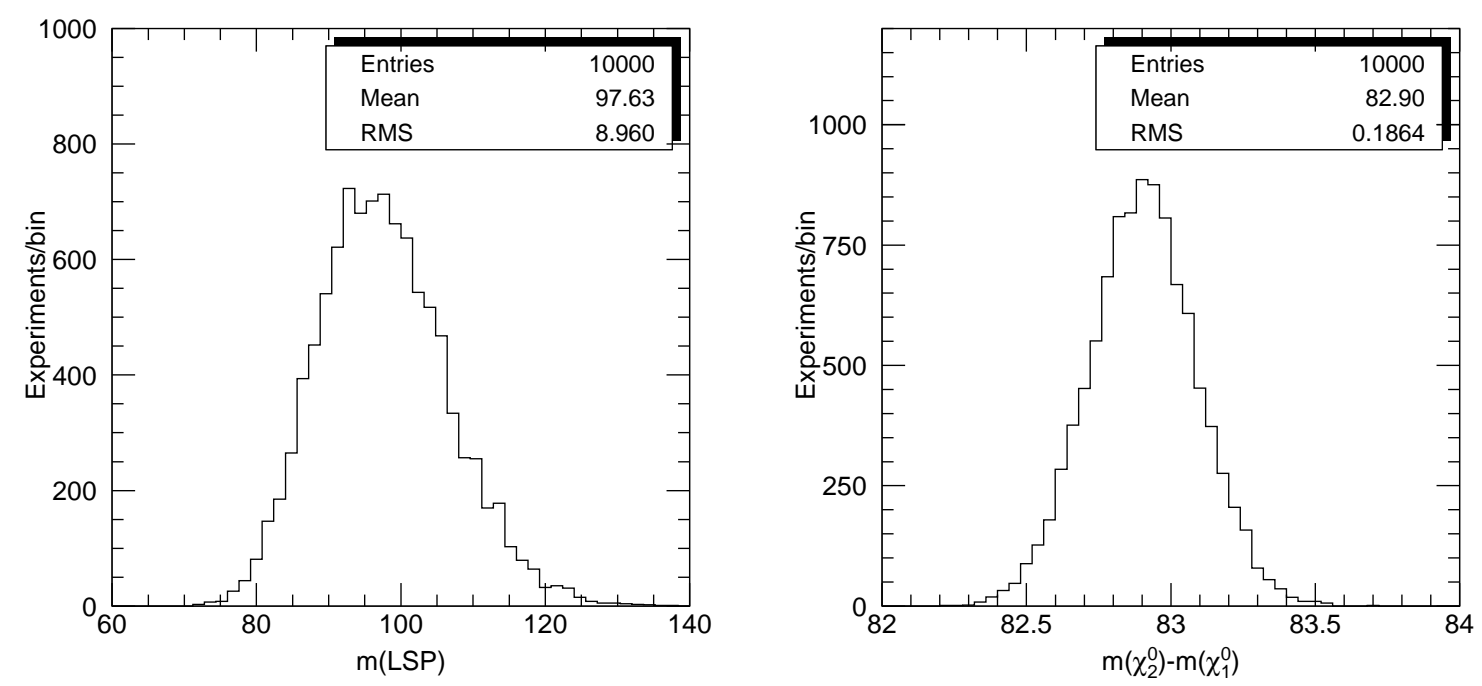

Figure 1: Distribution of the measured value of $m\left(\tilde{\chi}_{1}^{0}\right)$ (left) and of the difference $m\left(\tilde{\chi}_{2}^{0}\right)-$ $m\left(\tilde{\chi}_{1}^{0}\right)$ (right) for a set of Monte Carlo experiments, each corresponding to an integrated statistics of $300 \mathrm{fb}^{-1}$.

between masses. In particular, compared to the absolute error of $\sim 9 \mathrm{GeV}$ quoted above, the error on the difference $m\left(\tilde{\ell}_{R}\right)-m\left(\tilde{\chi}_{1}^{0}\right)$ is $\sim 200 \mathrm{MeV}$, as shown on the right side of Figure 1. thanks to the excellent precision of the measurement of the $m_{\ell \ell}^{\max }$ edge position. The calculated precision on $m\left(\tilde{\tau}_{1}\right)-m\left(\tilde{\chi}_{1}^{0}\right)$ is $\sim 2.5 \mathrm{GeV}$, scaling approximately linearly with the assumed error on $m_{\tau \tau}^{\max }$.

After extracting the mass measurements, the next step consists of constraining the neutralino sector, and calculating the components of the neutralino mixing matrix, which is a necessary ingredient in all calculations on the neutralino annihilation rate. Once this is fixed, we go on to constrain the slepton sector. The next step is understanding the constraints in the Higgs sector in order to evaluate possible contributions to the annihilation rate of Higgs exchange diagrams. By putting all of the information together we are finally able to give an estimate on the predictive power of the LHC data for a specific scenario in which the neutralino annihilation is dominated by processes involving sleptons.

\subsection{Constraints on the neutralino mass matrix}

Based on the expected LHC measurements, the masses of three neutralinos are measured: $\tilde{\chi}_{1}^{0}$, $\tilde{\chi}_{2}^{0}, \tilde{\chi}_{4}^{0}$. In the MSSM, assuming a $C P$-conserving scenario, the mass eigenstates $\chi_{i}^{0}(i=1,2,3,4)$ result from the mixing of the SUSY partners of the neutral gauge and Higgs bosons:

$$
\left(\tilde{B}, \tilde{W}^{3}, \tilde{H}_{1}^{0}, \tilde{H}_{2}^{0}\right)
$$


through a mixing matrix defined as:

$$
\mathcal{M}=\left(\begin{array}{cccc}
M_{1} & 0 & -m_{Z} \cos \beta s_{W} & m_{Z} \sin \beta s_{W} \\
0 & M_{2} & m_{Z} \cos \beta c_{W} & -m_{Z} \sin \beta c_{W} \\
-m_{Z} \cos \beta s_{W} & m_{Z} \cos \beta c_{W} & 0 & -\mu \\
m_{Z} \sin \beta s_{W} & -m_{Z} \sin \beta c_{W} & -\mu & 0
\end{array}\right)
$$

The above matrix is built out of four MSSM parameters: $M_{1}$ and $M_{2}$ are respectively the $\mathrm{U}(1)$ and $\mathrm{SU}(2)$ gaugino masses, $\mu$ is the Higgsino mass parameter, $\tan \beta=v_{2} / v_{1}$ is the ratio of the vacuum expectation values of the two Higgs doublets of the model. The additional parameters, $s_{W}$ and $c_{W}$, respectively the sine and cosine of the electroweak mixing angle $\theta_{W}$, and $m_{Z}$, the mass of the $Z$ boson are precisely known from measurements in the Standard Model. Given that we only have three input parameters in the three masses, we miss one parameter to fully solve the neutralino matrix. We take this parameter to be $\tan \beta$, as the other parameters relate directly to the neutralino masses. We show in Figure 2 the distribution of the reconstructed values of $Z_{11}$ and $Z_{13}$, for $\tan \beta=10$, where the composition of the $\tilde{\chi}_{1}^{0}$ is written as:

$$
\tilde{\chi}_{1}^{0}=Z_{11} \tilde{B}+Z_{12} \tilde{W}^{3}+Z_{13} \tilde{H}_{1}^{0}+Z_{14} \tilde{H}_{2}^{0}
$$

The spread from the experimental error is $0.02 \%$ for the bino component, and $1.5 \%$ for the
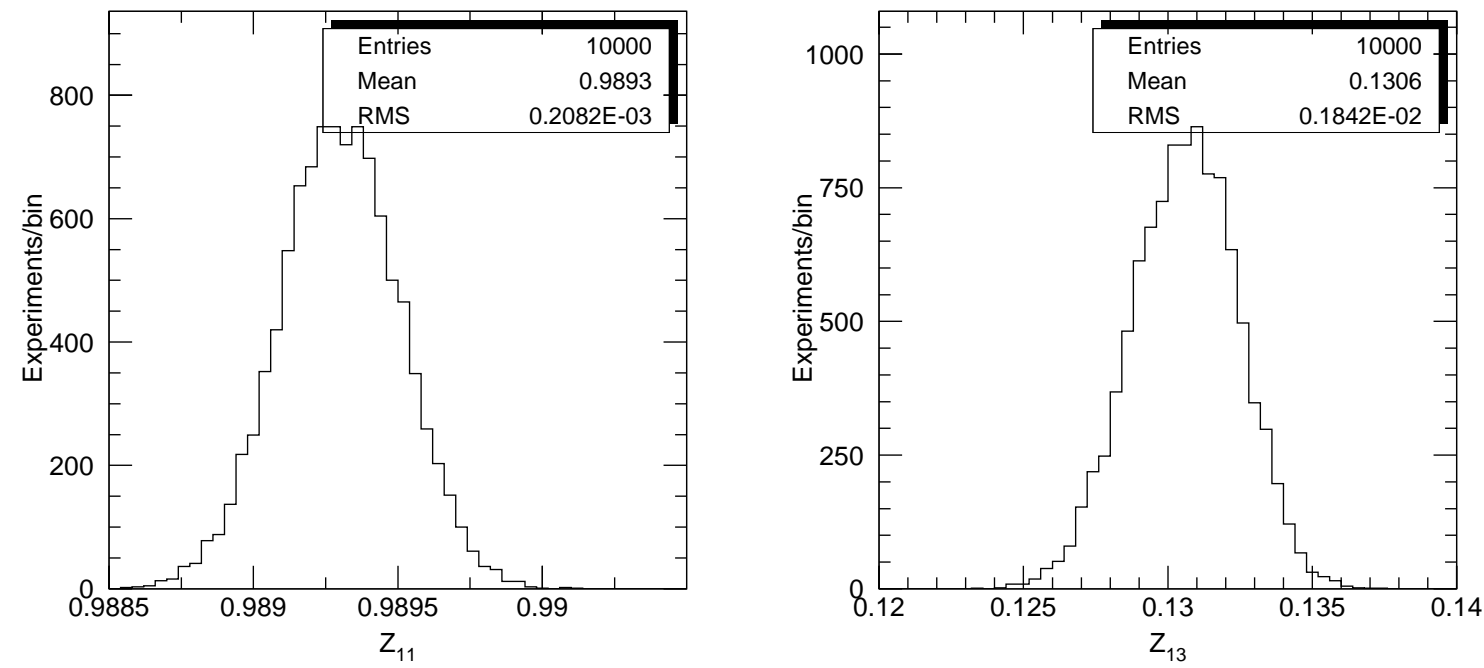

Figure 2: Distributions of the measured values of $Z_{11}$, the bino component of $\tilde{\chi}_{1}^{0}$ (left) and

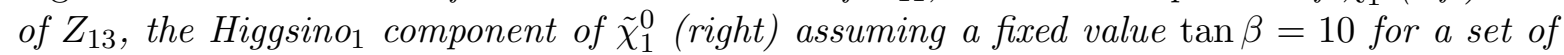
Monte Carlo experiments, each corresponding to an integrated statistics of $300 \mathrm{fb}^{-1}$.

other components. The dependence of the four components of the LSP on the assumed value of $\tan \beta$ is shown in Figure 3 , and for the assumed range $3-30$ is $0.8 \%$ for $Z_{11}$ and $\sim 15 \%$ for 

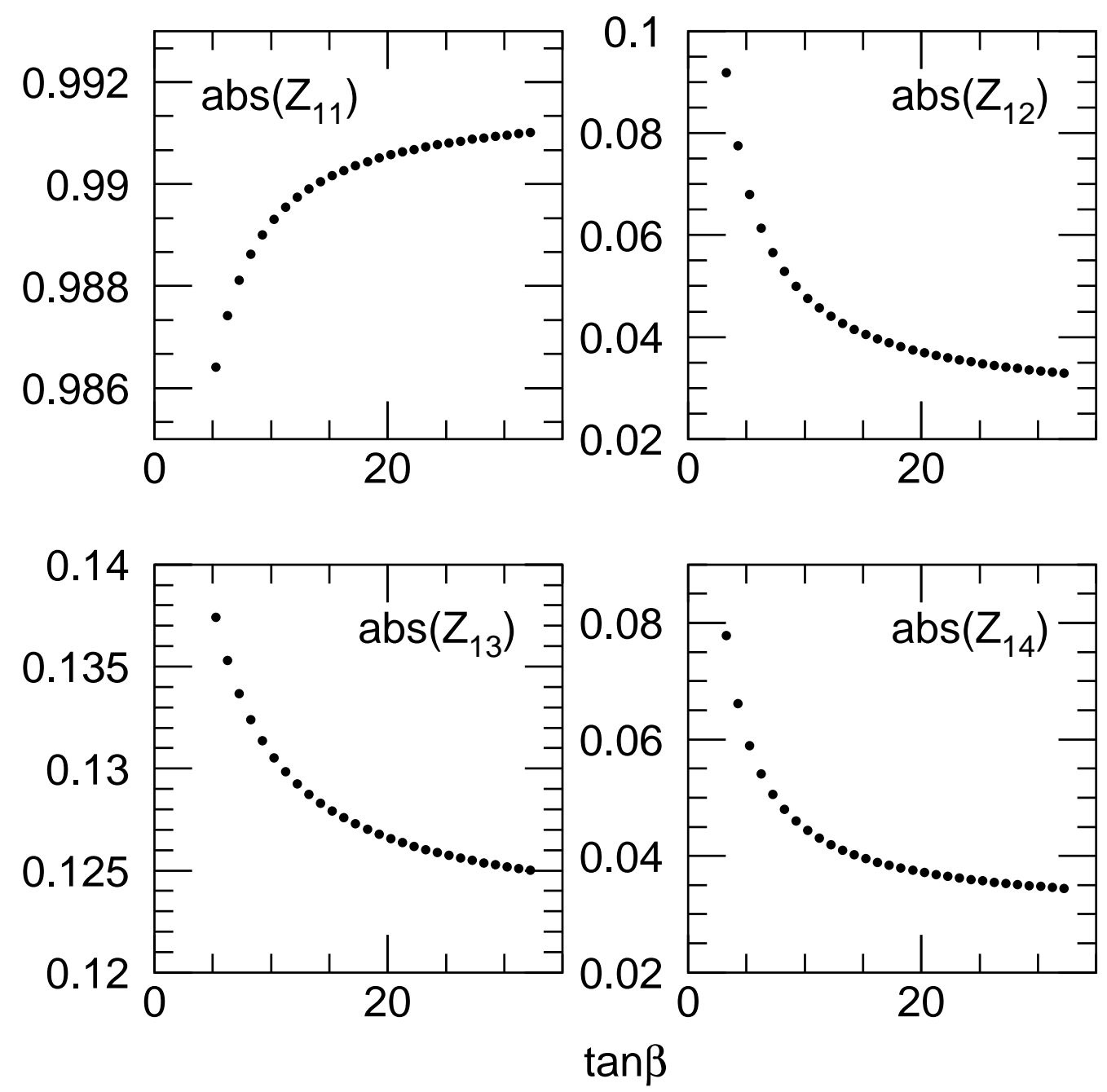

Figure 3: Average values of the four components of $\tilde{\chi}_{1}^{0}$ as a function of the assumed value of $\tan \beta$. The averages are performed on sets of Monte Carlo experiments, each corresponding to an integrated statistics of $300 \mathrm{fb}^{-1}$. 
$Z_{13}$. A much larger variation with $\tan \beta$ is observed for the smaller components $Z_{12}$ and $Z_{14}$. The sensitivity is higher for low values of $\tan \beta$. A first consideration from this measurement is that already at this stage the data tell us that the neutralino is an almost pure bino, with only a small Higgsino component. Annihilation channels involving the exchange of sfermions in the $t$-channel will dominate, unless the masses of the heavy Higgses are such that they resonantly enhance the relevant annihilation processes.

In the calculation above $M_{1}$ and $M_{2}$ are assumed as independent, since we are working on a general MSSM model. It is however customary in MSSM studies to assume that the ratio of the gaugino masses is equal to the ratio of the respective coupling constants, giving the relation $M_{2}=5 / 3 \tan ^{2} \theta_{W} M_{1}$. Under this assumption we can invert the gaugino mixing matrix using as constraints the mass differences $m\left(\tilde{\chi}_{2}^{0}\right)-m\left(\tilde{\chi}_{1}^{0}\right)$ and $m\left(\tilde{\chi}_{4}^{0}\right)-m\left(\tilde{\chi}_{1}^{0}\right)$ instead of the measured masses, and extract the values of $M_{2}$ and $\mu$, always for fixed $\tan \beta$. From these one can in turn calculate the values of the gaugino masses. In this case the absolute scale is fixed in a much more precise way, and the resolution on $m\left(\tilde{\chi}_{1}^{0}\right)$ is $\sim 250 \mathrm{MeV}$. We will keep as a baseline for our study the completely unconstrained model, but we will also show the results when the gaugino mass unification is assumed.

\subsection{Constraints on the slepton sector}

Once the neutralino mass is extracted, the slepton sector can be considered. No mixing is assumed here in the selectron and smuon case. It was shown in 21] that the difference in mass of the electron and muon should result in different mixings for the selectron and the smuon, resulting for the considered model point in a small mass difference and in a $\sim 8 \%$ difference between $B R\left(\tilde{\chi}_{2}^{0} \rightarrow \tilde{e}_{R} e\right)$ and $B R\left(\tilde{\chi}_{2}^{0} \rightarrow \tilde{\mu}_{R} \mu\right)$. We assume for this analysis that no mixing takes place in the selectron and smuon sector.

Due to the hypercharge difference, the LSP pair annihilation cross section through $t$ channel exchange of $\tilde{\ell}_{R}$ is 16 times bigger than that for $\tilde{\ell}_{L}$ exchange when $m\left(\tilde{\ell}_{R}\right)_{R}=m\left(\tilde{\ell}_{L}\right)$ and the LSP is dominantly bino. Therefore if the chirality of the slepton observed in the $\tilde{\chi}_{2}^{0}$ decay chain can not be determined, this could cause a very large uncertainty in the relic density calculation. The issue is however discussed in [21, where it is shown that the asymmetry distribution proposed in [22] is sensitive to the chirality structure of the slepton. Those studies were performed for the SPS1a point, so their results can be safely extended to the model addressed in the present study, and we can assume for the present study that the chirality of the lighter slepton can be determined.

In the stau sector, the ratio $B R\left(\tilde{\chi}_{2}^{0} \rightarrow \tilde{\ell}_{R} \ell\right) / B R\left(\tilde{\chi}_{2}^{0} \rightarrow \tilde{\tau}_{1} \tau\right)$ is a function of the neutralino mixing matrix, of $m\left(\tilde{\tau}_{1}\right), m\left(\tilde{\chi}_{2}^{0}\right), \tan \beta$ and $\theta_{\tau}$, the mixing angle between $\tilde{\tau}_{R}$ and $\tilde{\tau}_{L}$. By assuming a given value for $\tan \beta$ we can therefore from this measurement extract the value of $\theta_{\tau}$. The distribution of the measured $\theta_{\tau}$, as well as the dependence on the input $\tan \beta$ value are are shown in Figure 4. The experimental uncertainty is $\sim 2 \%$, whereas $\theta_{\tau}$ varies by $\sim 35 \%$ over the considered $\tan \beta$ range.

The only missing parameter in order to be able to calculate the neutralino annihilation processes involving sleptons is the mass of the $\tilde{\tau}_{2}$ slepton. Natural bounds can be imposed to this mass. First of all we must have $m\left(\tilde{\tau}_{2}\right)>m\left(\tilde{\chi}_{2}^{0}\right)-m(\tau)$, otherwise the $\tilde{\tau}_{2}$ contribution would likely be observed in the $\tau \tau$ edge from the $\tilde{\chi}_{2}^{0}$ decay. Second, from $m\left(\tilde{\tau}_{1}\right), \theta_{\tau}, \tan \beta$ and $m\left(\tilde{\tau}_{2}\right)$ the value for the trilinear coupling $A_{\tau}$ can be calculated. Large values of the $A_{\tau}$ parameter could induce charge breaking minima (CCB) due to the vacuum expectation values 

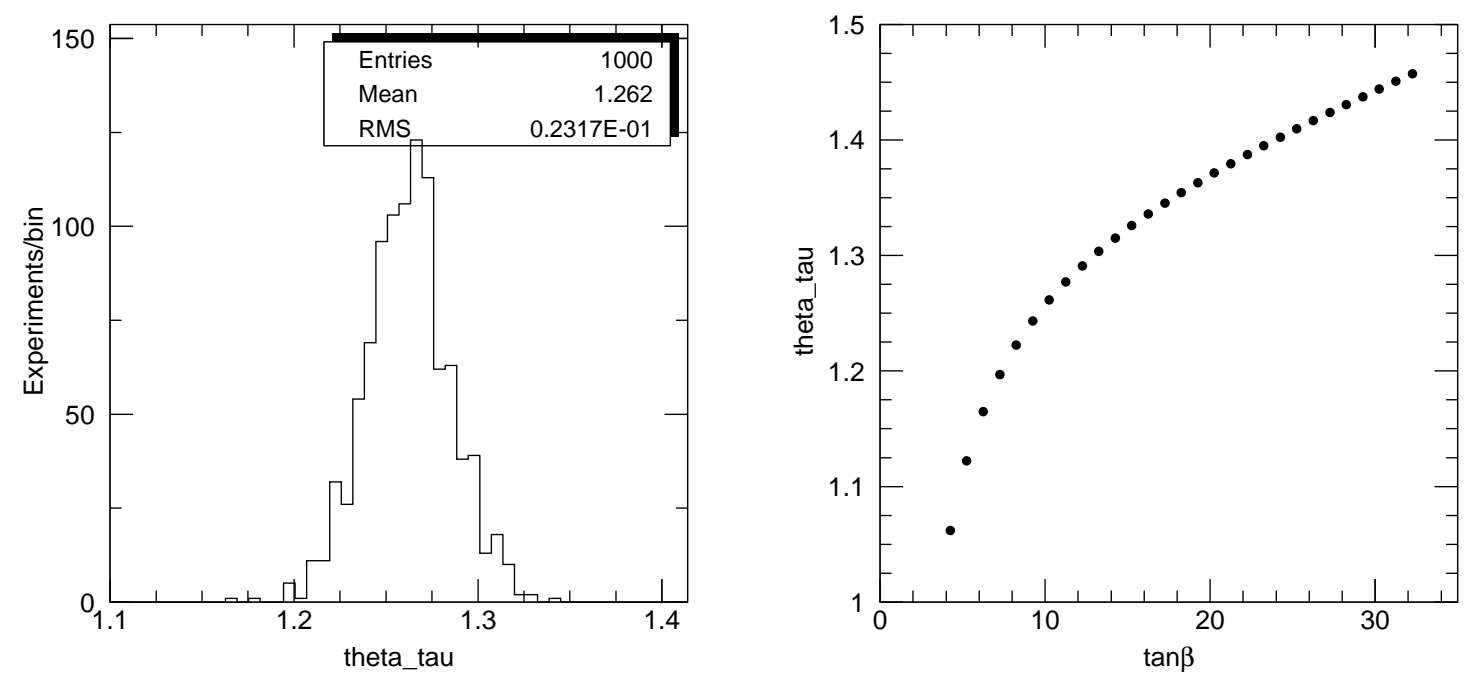

Figure 4: Left: distribution of the measured value of $\theta_{\tau}$, the $\tilde{\tau}$ mixing angle assuming a fixed value $\tan \beta=10$ for a set of Monte Carlo experiments, each corresponding to an integrated statistics of $300 \mathrm{fb}^{-1}$. Right: average values of $\theta_{\tau}$, as a function of the assumed value of $\tan \beta$. The averages are performed on sets of Monte Carlo experiments, each corresponding to an integrated statistics of $300 \mathrm{fb}^{-1}$.

of the charged $\tau$ scalars. Typical constraints on $A_{\tau}$ from these considerations [23] would give an upper limit on $A_{\tau}$ of $\sim 500 \mathrm{GeV}$, very near to the actual $A_{\tau}$ value for the considered model. The conditions on CCB minima resulting in constraints on the MSSM are in general neither necessary nor sufficient to give an acceptable vacuum structure to the theory, and it has been suggested [24] that the constraint from 23] be relaxed through an empirical multiplicative factor. Following these considerations we conservatively fix here a limit of $5 \mathrm{TeV}$ to $A_{\tau}$, which, in the considered model, for $\tan \beta=10$ corresponds to a limit $m\left(\tilde{\tau}_{2}\right)<250 \mathrm{GeV}$.

\subsection{Constraints from the Higgs sector}

As discussed in the previous sections, the measurements considered up to now do not allow us to constrain the $\tan \beta$ parameter. The main constraints come from the Higgs sector. In particular, the measurement of the mass and of the production rate of one of the heavy Higgs would define a confidence region in the canonical $m(A)-\tan \beta$ plane. The region where heavy Higgses can be discovered through their decays to Standard Model particles are shown in Figure [5] from [14.

The plot was obtained on specific assumptions on the SUSY spectrum, i.e. a SUSY mass scale $M_{S U S Y}$ of $1 \mathrm{TeV}$, and maximal mixing, i.e. $\mu \ll M_{S U S Y}$ and the trilinear couplings $A=\sqrt{6} M_{S U S Y}$. These assumptions mostly affect the considerations related to the light Higgs, and only lightly affect the discovery region for $\mathrm{H} / \mathrm{A}$ and $H^{+}$. Given that the mixing in the chosen model is less than maximal $\left(A_{t}=-450 \mathrm{GeV}\right)$, the limit on $\tan \beta, \tan \beta>3$ 


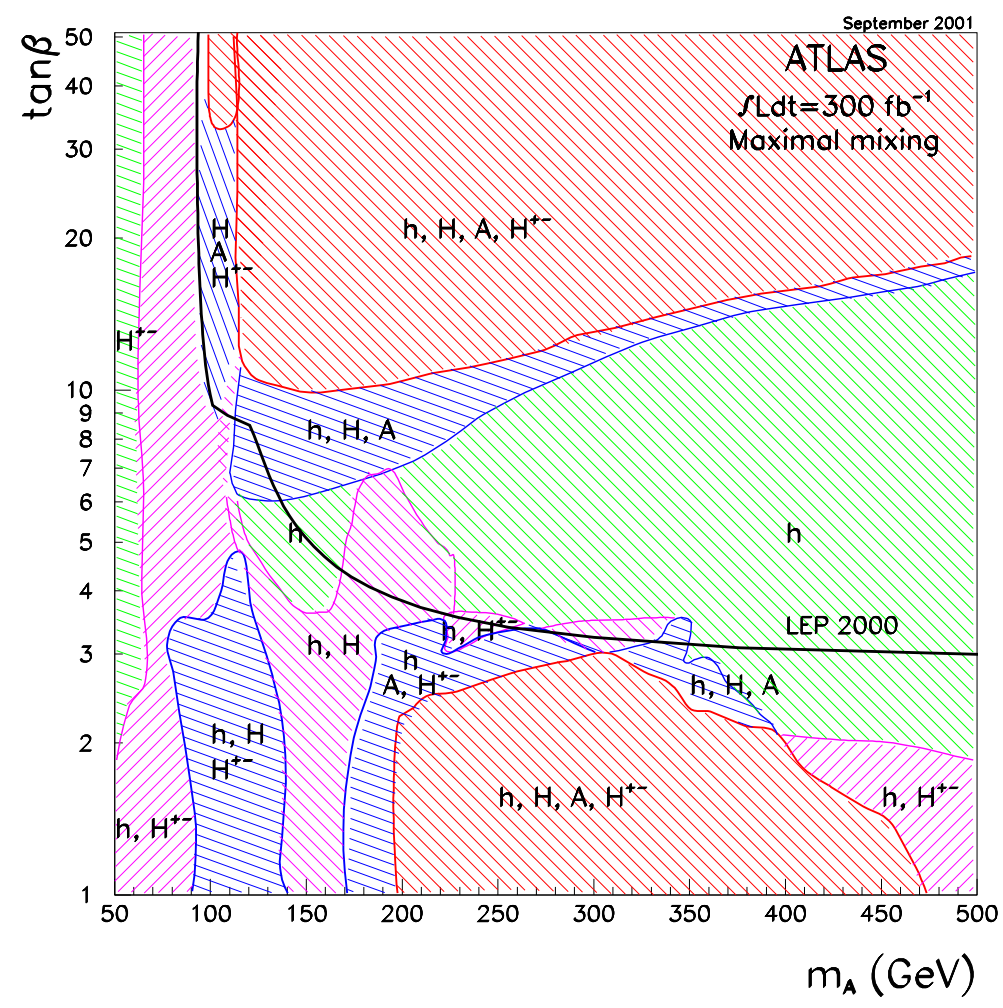

Figure 5: Reach of the ATLAS experiment in the $m(A)-\tan \beta$ plane for an integrated luminosity of $300 \mathrm{fb}^{-1}$. For each region in the plane, the detectable Higgs bosons are marked.

shown in Figure [5] can be used as lower limit of the $\tan \beta$ range considered in this analysis. The considered model, corresponding to $m_{A}=424 \mathrm{GeV}$ and $\tan \beta=10$ lies outside of the region where a heavy Higgs can be discovered, we will limit our analysis to the complement of this region in the $m(A)-\tan \beta$ plane.

A stronger constraint can be obtained by the fact that the light Higgs $h$ will be discovered at the LHC and its mass measured. From this measurement a confidence band in the $m(A)-\tan \beta$ plane can be defined.

Finally, since the SUSY spectrum is largely known, we can investigate whether the heavy Higgses can be detected either a) in the cascade decay of a sparticle, or b) through their decay into a sparticle, or a bound can be extracted from the non-observation.

For the cascade decays, no heavy Higgs appears in the decay chains, as all the decays of neutralinos/charginos into heavy Higgses are kinematically closed. We can however investigate up to which Higgs masses these decays would be open. The best candidates would be the decays: $\tilde{\chi}_{4(3)}^{0} \rightarrow \tilde{\chi}_{1(2)}^{0} A / H, \tilde{\chi}_{2}^{ \pm} \rightarrow \tilde{\chi}_{1}^{ \pm} A / H$ with subsequent decays of the $\mathrm{A} / \mathrm{H}$ into $b \bar{b}$. These decays have been studied in [25] where it is shown that in favourable conditions a peak in $b \bar{b}$ distribution can be observed. The kinematic limits in the considered model are: 
- $m(A / H) \leq 315 \mathrm{GeV}$ for $\tilde{\chi}_{4(3)}^{0} \rightarrow \tilde{\chi}_{1}^{0} A / H$

- $m(A / H) \leq 230 \mathrm{GeV}$ for $\tilde{\chi}_{4(3)}^{0} \rightarrow \tilde{\chi}_{2}^{0} A / H$ and $\tilde{\chi}_{2}^{ \pm} \rightarrow \tilde{\chi}_{1}^{ \pm} A / H$.

We evaluated the number of events in which a $H / A \rightarrow b \bar{b}$ decay is produced in the cascade decay of squarks and gluinos by generating with HERWIG 26] a sample of events for the chosen model, and computing the fraction of events containing either of $\tilde{\chi}_{3}^{0}, \tilde{\chi}_{4}^{0}, \tilde{\chi}_{2}^{ \pm}$. We thereafter computed with ISASUSY [1] the BR of the charginos/neutralinos for the considered model and different values of $m(A)$, and convoluted the results with the total SUSY cross-section calculated at NLO with PROSPINO [27]. The expected numbers of events as a function of $m(A)$ for an integrated luminosity of $300 \mathrm{fb}^{-1}$ are shown in Figure 6. The curve clearly shows the drop in number of events when the decays to $\tilde{\chi}_{2}^{0}$ and $\tilde{\chi}_{1}^{ \pm}$become kinematically inaccessible, and the fast decrease to zero after $300 \mathrm{GeV}$. For $m(A)=300 \mathrm{GeV}$, the number

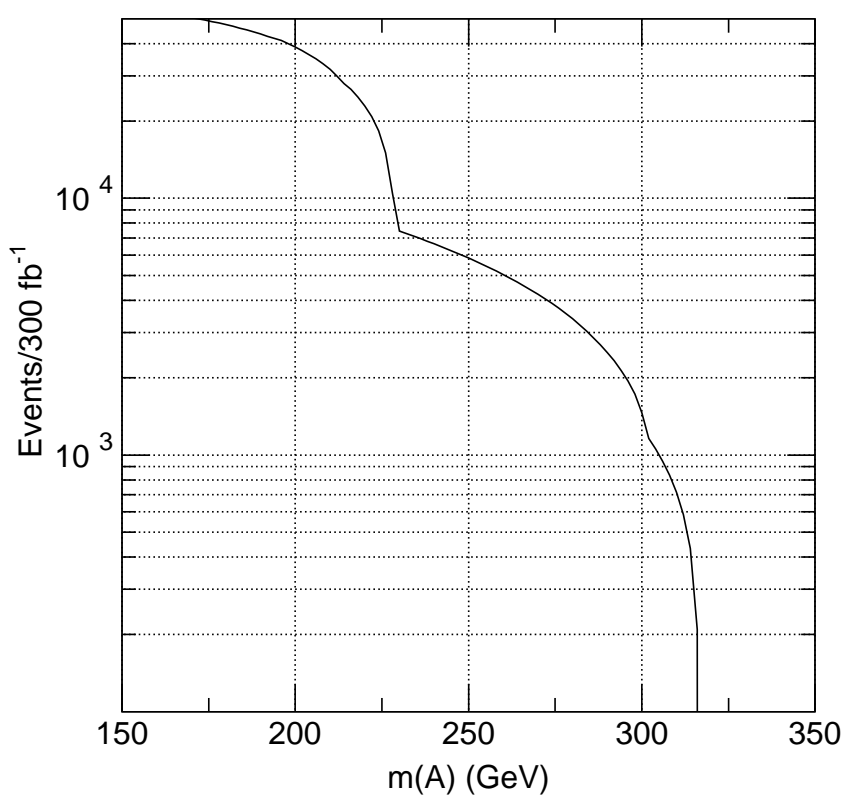

Figure 6: Number of expected events containing a $H / A \rightarrow b \bar{b}$ decay in the cascade of squarks or gluinos, as a function of $m(A)$. The assumed integrated luminosity is $300 \mathrm{fb}^{-1}$

of events contributing to the $b \bar{b}$ peak is $\sim 1500$. It should therefore be possible to put a lower bound on the heavy Higgs mass of approximately $300 \mathrm{GeV}$. In order to verify whether this is actually possible, a detailed experimental analysis is needed, outside of the scope of the present study.

For the SUSY decays to heavy Higgses, two channels have been identified as particularly promising, and have been the subject of detailed experimental analyses [14, 28, 29, 30]:

- $H^{ \pm} \rightarrow \tilde{\chi}_{i}^{0} \tilde{\chi}_{1}^{ \pm} \rightarrow 3 \ell+E_{T}^{m i s s}$ 
- $A / H \rightarrow \tilde{\chi}_{2}^{0} \tilde{\chi}_{2}^{0} \rightarrow 4 \ell+E_{T}^{m i s s}$

The first channel is hopeless for the considered model, as the value of the branching ratio for the charged Higgs decay to three leptons through a chargino-neutralino pair is of order a few $10^{-6}$.

For the channel $A / H \rightarrow \tilde{\chi}_{2}^{0} \tilde{\chi}_{2}^{0}$, the total BR to 4 leptons through the second neutralino is $1.36 \times 10^{-4}\left(0.46 \times 10^{-4}\right)$ respectively for the $A(H)$. We considered the $A$ and $H$ production both through gluon fusion, and in association with two $b$ quarks, based on the available NLO calculations [31, 32]. A total of 40 events per experiment is produced for an integrated luminosity of $300 \mathrm{fb}^{-1}$. The typical analysis efficiency for this signal is $30 \%$, with the reduction of the SM background to negligible levels. The dominant SUSY background can be further reduced with respect to previous analyses by the possibility of performing a full reconstruction of the $\mathrm{A} / \mathrm{H}$ mass peak, as discussed in 33 . It is therefore thinkable that the heavy Higgs can be discovered in this channel. However, given the small number of events considered, a very careful background study, taking into account the details of the detector performance would be required.

In conclusion, three scenarios can be envisaged:

- A scenario in which the only constraints on the $m(A)$ - $\tan \beta$ plane are provided by the measurement of the light Higgs $h$, and by the non-discovery of the heavy Higgses in their Standard Model decay modes

- A scenario in which a lower limit of approximately $300 \mathrm{GeV}$ on the $H / A$ mass can be set by the non-observation of the heavy Higgses in the SUSY cascade decays.

- A scenario in which the $H / A$ is discovered in its $\tilde{\chi}_{2}^{0} \tilde{\chi}_{2}^{0}$ decay mode.

In the following we will evaluate the constraints on $\Omega_{\chi} h^{2}$ taking into account all the three scenarios.

\section{Calculation of the relic density}

We can at this point calculate using the micrOMEGAs program [12] the LSP relic density $\Omega_{\chi} h^{2}$ for each of the Monte Carlo experiments defined in Section 2, The parameters derived from the measurements given in Table 3 used to create the Monte Carlo experiments are the masses of $\tilde{\chi}_{1}^{0}, \tilde{\chi}_{2}^{0}, \tilde{\chi}_{4}^{0}, \tilde{\ell}_{R}, \tilde{\ell}_{L}$, and $\tilde{\tau}_{1}$ and the stau mixing angle $\theta_{\tau}$. From these, for each experiment the soft MSSM parameters to give as input to micrOMEGAs are calculated. The nominal values are assumed for all the other MSSM parameters, in particular $m(A), \tan \beta$ and $m\left(\tilde{\tau}_{2}\right)$.

The resulting distributions of the calculated $\Omega_{\chi} h^{2}$ are given in Figure 7 for two values of the assumed uncertainty on the position of the $\tau \tau$ edge, respectively $5 \mathrm{GeV}$ and $0.5 \mathrm{GeV}$. The error is respectively $\sim 20 \%(10 \%)$ for an uncertainty of $5(0.5) \mathrm{GeV}$. This uncertainty is quoted in Table 3 as $5 \mathrm{GeV}$, and translates to an uncertainty on the mass difference $m\left(\tilde{\tau}_{1}\right)-m\left(\tilde{\chi}_{1}^{0}\right)$ of $2.5 \mathrm{GeV}$. The measurement precision on this difference has been found in 34 to be the dominant factor in the determination of the relic density. No detailed study is available on the precision with which the LHC experiments can define the $\tau \tau$ edge, and $5 \mathrm{GeV}$ was quoted in [16] as a conservative upper limit on this figure. It is therefore interesting to treat this uncertainty as a parameter of the analysis. We have therefore re-evaluated the neutralino 
relic density as a function of the error on the $\tau \tau$ edge position. The results are shown as full circles in Figure 8. The overall uncertainty depends on the $\tau \tau$ measurement as long as the uncertainty is above $\sim 1 \mathrm{GeV}$, which therefore should be taken as the goal for the systematic control to be achieved on the measurement of this variable.

The remaining uncertainty of $10 \%$ is determined by the $\sim 10 \%$ error on the $\tilde{\chi}_{1}^{0}$ mass, as can be seen in Figure 9, produced for $\sigma(m(\tau \tau))=1 \mathrm{GeV}$, where the predicted relic density is shown as a function of the measured $\tilde{\chi}_{1}^{0}$ mass. If we assume gaugino mass universality, as discussed in Section 3, the uncertainty on the $\tilde{\chi}_{1}^{0}$ mass is reduced to $\sim 250 \mathrm{MeV}$. We show as full squares in Figure 8 the evolution of the uncertainty as a function of the error on the $\tau \tau$ edge position under this assumption. For an uncertainty on $m(\tau \tau)$ of $1 \mathrm{GeV}$, the measurement error is reduced to $\sim 4 \%$.

We have also studied the effect of changing the systematic uncertainty on the measurement of $B R\left(\tilde{\chi}_{2}^{0} \rightarrow \tilde{\ell}_{R} \ell\right) / B R\left(\tilde{\chi}_{2}^{0} \rightarrow \tilde{\tau}_{1} \tau\right)$ from $10 \%$ to $1 \%$. The effect has been shown to be negligible as compared to the other uncertainty sources.
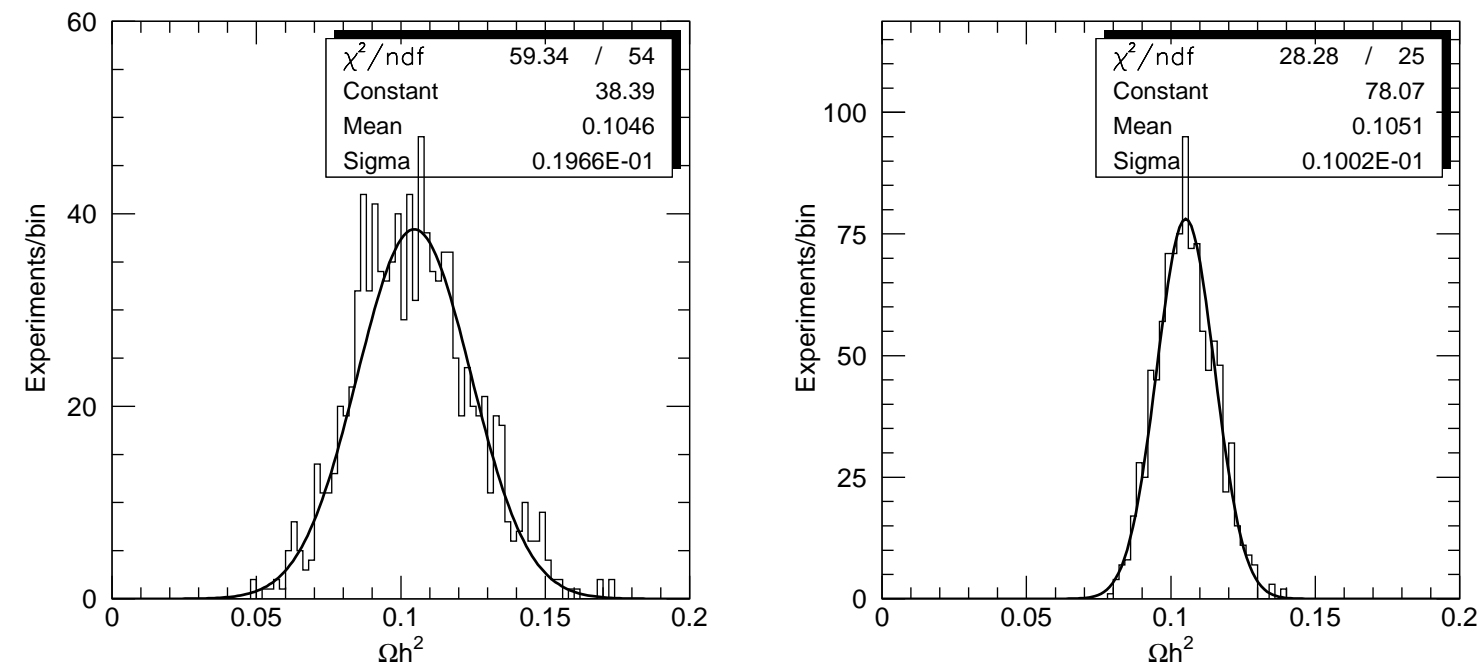

Figure 7: Distributions of the predicted relic density $\Omega_{\chi} h^{2}$ incorporating the experimental errors. The distributions are shown for an assumed error on the $\tau \tau$ edge respectively of $5 \mathrm{GeV}$ (left) and $0.5 \mathrm{GeV}$ (right).

We further studied in detail the dependence of the relic density prediction on the parameters which are only very loosely constrained (if at all) by the LHC data. The squark and gluino masses, will be measured with a precision of a few percent at the LHC, but for the masses considered, the relic density is essentially independent from their value. The only loophole could be a light stop. We have studied the dependence of the predicted relic density on the mass of the stop. We found that a light stop would only contribute to the relic density if its mass were below $\sim 140 \mathrm{GeV}$. A detailed study on a similar model point, given in [35, shows that the lighter stop, which has a mass of $\sim 400 \mathrm{GeV}$, can be observed in the cascade decays 


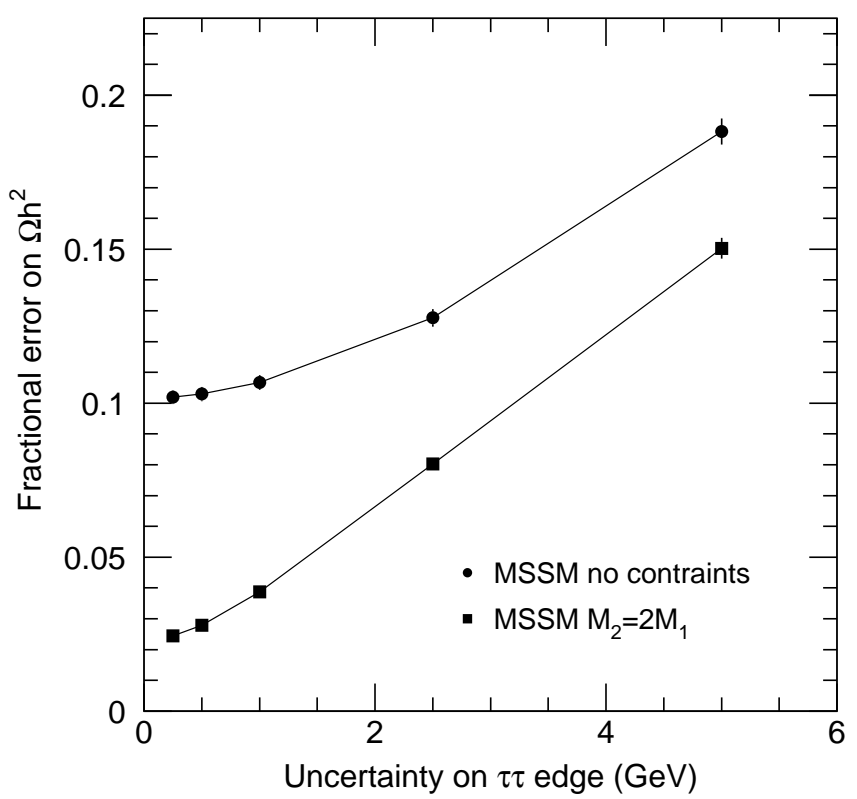

Figure 8: Fractional uncertainty on the predicted relic density $\Omega_{\chi} h^{2}$ from the experimental measurement, as a function of the assumed uncertainty on the position of the $\tau \tau$ edge respectively for the completely unconstrained MSSM (black circles), and under the assumption of gaugino mass unification (black squares).

of the gluino, and it is possible to build kinematic edges which can be used to constrain its mass. We can therefore assume that for the considered model it will be possible to exclude a significant stop coannihilation contribution to the relic density. The main loosely constrained parameters which influence the relic density calculation are therefore: $\tan \beta, m(A)$, and $m\left(\tilde{\tau}_{2}\right)$. We have therefore varied in turn $m\left(\tilde{\tau}_{2}\right), \tan \beta, m(A)$, while the other soft parameters going in input to micrOMEGAs are recalculated in such a way that the masses of the sparticles which can be measured experimentally are kept fixed at their nominal value.

For this exercise we have assumed the minimum constraint in the $m(A)-\tan \beta$ plane discussed above, assuming a lower bound on $\tan \beta$ of 3 , and vetoing the regions where the heavy Higgs can be discovered by the LHC experiments at $5 \sigma$ through their $S M$ decays. We have moreover imposed the loose bounds on $m\left(\tilde{\tau}_{2}\right)$ discussed above. The dependencies on the different parameters are shown in Figures 10, 11] and 12,

It is clear from these figures that if we can not extract from the data any direct information on the mass of the heavy Higgses, only an upper limit on the neutralino relic density can be given. If it is possible to exclude heavy Higgs masses lighter than $300 \mathrm{GeV}$ through the peak search in the $b \bar{b}$ mass distribution, the spread in relic density measurement is of order $1 \%$, and is strongly dependent on the experimental value of the lower limit on the mass of the heavy Higgs. If the heavy Higgses are directly observable through their decays to SUSY particles, 


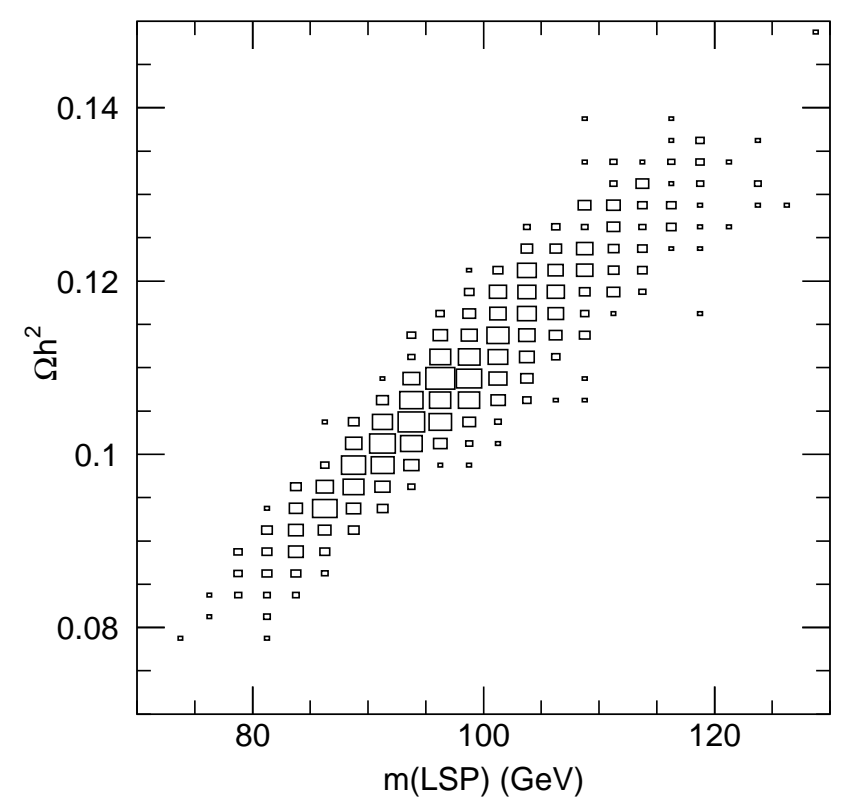

Figure 9: Value of the predicted relic density $\Omega_{\chi} h^{2}$ as a function of the measured $\tilde{\chi}_{1}^{0}$ mass.

the Higgs mass is fixed, and its experimental uncertainty does not contribute to the error on the relic density prediction, as for high Higgs masses the contribution of channels involving Higgses to the neutralino annihilation is negligible.

In this case the dominant contribution to the uncertainty will come from the poorly constrained value of $\tan \beta$. In the interval allowed by the non-observation of the $S M$ decays of the Higgs, the relic density varies by $\sim 11 \%$, as shown in the right side of Figure 11. It is interesting to disentangle the contributions of the different annihilation processes to the variation. In the right side of Figure 11 we show the annihilation cross-sections (in units of their contribution to $1 / \Omega)$. For the different processes. The spread is dominated by the $\tilde{\chi}_{1}^{0} \tilde{\chi}_{1}^{0} \rightarrow \tau^{+} \tau^{-}$process. The reason is that for each value of $\tan \beta$ we recalculate the soft parameters in such a way that the sparticle masses, and the branching ratios which are measured experimentally are kept constant. Therefore the composition of the $\tilde{\chi}_{1}^{0}$ and the value of $\theta_{\tau}$ vary as shown in Figures 3 producing the dependency observed in the full line in the left side of Figure 11]

An additional uncertainty will come from the value of $m\left(\tilde{\tau}_{2}\right)$, which contributes a $\sim 7 \%$ spread to the result, as shown in Figure 12. This is because the $\tilde{\tau}_{2}$ exchange contribution is opposite to the $\tilde{\tau}_{1}$ contribution. The cancellation appears in the $s$-wave part of the pair annihilation cross section, which is chirality suppressed. In the limit where the Higgsino component of the lightest neutralino can be ignored, the $\tilde{\tau}_{i}$ contribution to the amplitude can be expressed as

$$
M(\text { swave }) \propto \sin \theta_{\tau} \cos \theta_{\tau}\left[1 /\left(1+m_{\tilde{\tau}_{1}}^{2} / m_{\tilde{\chi}_{1}^{0}}^{2}\right)-1 /\left(1+m_{\tilde{\tau}_{2}}^{2} / m_{\tilde{\chi}_{1}^{0}}^{2}\right)\right] Z_{11}^{2}
$$



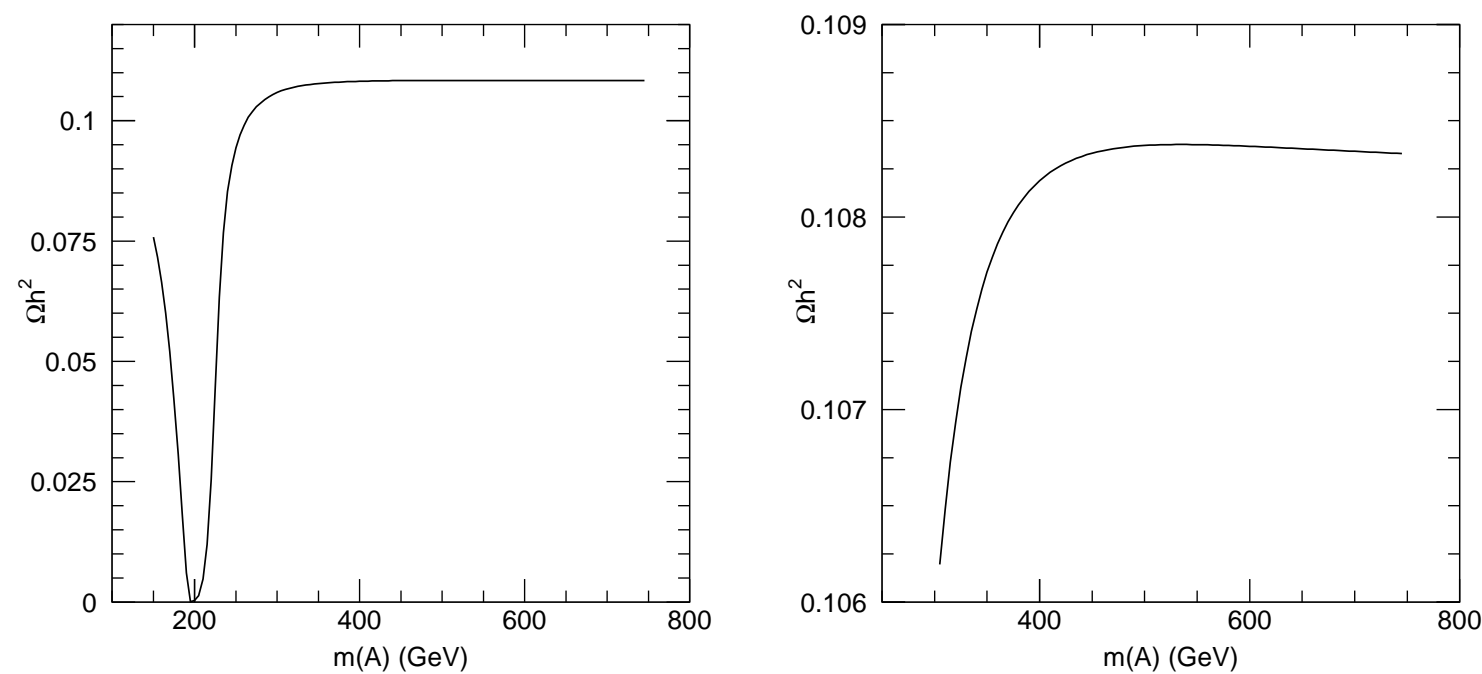

Figure 10: Dependency of the relic density $\Omega_{\chi} h^{2}$ from the value of the pseudoscalar Higgs mass $m(A)$. The dependency is shown respectively for the whole $m(A)$ mass range (left), and for $m(A)>300 \mathrm{GeV}$ (right).

yielding the observed dependence of the annihilation cross-section on $m\left(\tilde{\tau}_{2}\right)$. The mixing angle $\theta_{\tau}$ is kept constant by construction in Figure 12 because we use the ratio of decay widths $\Gamma\left(\tilde{\chi}_{2}^{0} \rightarrow e e \tilde{\chi}_{1}^{0}\right) / \Gamma\left(\tilde{\chi}_{2}^{0} \rightarrow \tau \tau \tilde{\chi}_{1}^{0}\right)$ as the constraint on $\theta_{\tau}$.

\section{Discussion}

In the above discussion we have considered a 'bulk region' SUSY model in which $\tilde{\chi}_{1}^{0}$ annihilation in the early universe is dominated by diagrams involving light sleptons. This model lies within the mSUGRA sub-space of the full MSSM parameter space, however we have shown that it is possible to set useful constraints on the neutralino relic density without making special assumptions on the behaviour of the theory at high scale.

It is interesting to consider whether this result applies more generally to SUSY models characterised by dominant light slepton $\tilde{\chi}_{1}^{0}$ annihilation. The starting point in the analysis is the possibility of isolating the decay chains $\tilde{q}_{L} \rightarrow \tilde{\chi}_{2}^{0} q \rightarrow \tilde{\ell}_{r}^{ \pm} \ell^{\mp} q \rightarrow \ell^{ \pm} \ell^{\mp} q \tilde{q}_{L} \rightarrow \tilde{\chi}_{2}^{0} q \rightarrow$ $\tilde{\tau}_{1}^{ \pm} \tau^{\mp} q \rightarrow \tau^{ \pm} \tau^{\mp} q$ with sufficient statistics to be able to observe the kinematic edges providing the mass measurement. One therefore requires the mass hierarchy $m\left(\tilde{q}_{L}\right)>m\left(\tilde{\chi}_{2}^{0}\right)>m\left(\tilde{\ell}_{R}\right)$ and equivalently for the $\tilde{\tau}_{1}$. The former condition on $m\left(\tilde{\chi}_{2}^{0}\right)$ and $m\left(\tilde{\ell}_{R}\right)$ is generally satisfied in the light slepton annihilation region, and if stau annihilation is to be relevant so must the latter. To avoid significant squark co-annihilation $m\left(\tilde{q}_{L}\right)$ must by definition be somewhat larger than the mass scale of the sleptons and lightest neutralino. It is therefore likely that at least the necessary decay chain(s) will occur, although the mass differences may be sufficiently small that one or more of the kinematic end-points are unobservable. 

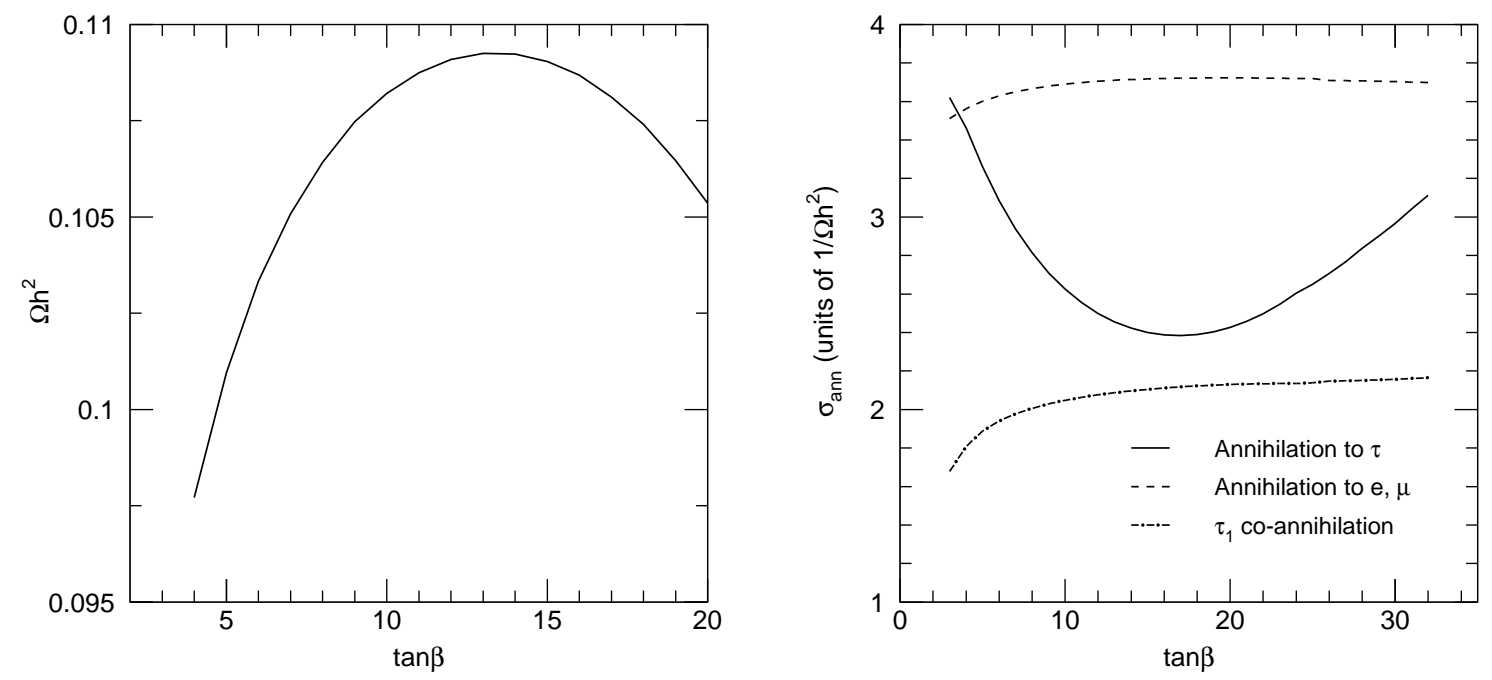

Figure 11: Left: Dependency of the relic density $\Omega_{\chi} h^{2}$ from the value of $\tan \beta$. Right: Dependency of the annihilation cross-section for different processes, respectively $\tilde{\chi}_{1}^{0} \tilde{\chi}_{1}^{0} \rightarrow \tau^{+} \tau^{-}$ (full line), $\tilde{\chi}_{1}^{0} \tilde{\chi}_{1}^{0} \rightarrow \ell^{+} \ell^{-}$(dashed line) and $\tilde{\chi}_{1}^{0} \tilde{\tau}_{1} \rightarrow Z / A \tau$ (dot-dashed line). The units are contributions to $1 / \Omega h^{2}$.

In order to obtain sufficient statistics of events containing the above decay chain, $m\left(\tilde{q}_{L}\right)$ must be $\lesssim 1 \mathrm{TeV}$, and the $\tilde{\chi}_{2}^{0}$ must have a large Wino component to couple to $\tilde{q}_{L}$. Moreover, the mass of the left handed component of $\tilde{\tau}_{1}$ should not be too large, to avoid the decay $\tilde{\chi}_{2}^{0} \rightarrow \tilde{\tau}_{1} \tau$ saturating the $\tilde{\chi}_{2}^{0}$ branching ratio and thus killing the lepton signature. From the experimental point of view, we need to add the requirement that the masses of the involved sleptons are not too near to the mass of either neutralino. A further essential ingredient in the reconstruction of the neutralino mass matrix is the possibility of measuring the mass of the $\tilde{\chi}_{4}^{0}$. This implies non-negligible gaugino components in the $\tilde{\chi}_{4}^{0}$, the appropriate mass hierarchy and typically the knowledge of the $\tilde{\ell}_{L}$ mass, either through its direct production or through it appearance in a cascade decay [36]. Such requirements on the neutralino and squark sectors need not be satisfied in the light slepton $\tilde{\chi}_{1}^{0}$ annihilation region; if this were indeed the case the analysis would be more challenging.

Finally, we also require that it be possible to constrain alternative contributions to the annihilation cross-section through exclusion of the required values of $m_{A}$ and $m\left(\tilde{t}_{1}\right)$ by direct measurement or non-observation of a signal in an appropriate channel. If such constraints can not be obtained then the obtained value for $\Omega h^{2}$ is merely an upper limit.

We may consider also whether the techniques described here may be applied to SUSY models in which alternative annihilation mechanisms dominate. In general in order to obtain an estimate of $\Omega h^{2}$ it is necessary not just to measure the dominant contributions to the $\tilde{\chi}_{1}^{0}$ annihilation cross-section but also to constrain all other possible contributions. In any such analysis therefore the masses and mixings of the neutralino, light slepton, stau, stop and 


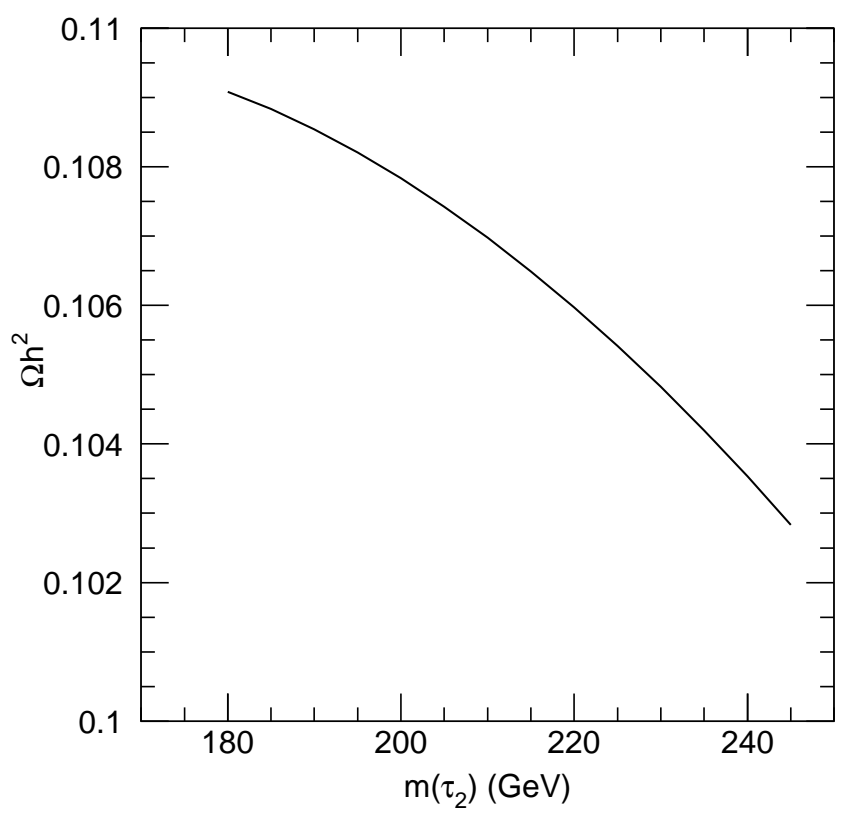

Figure 12: Dependency of the relic density $\Omega_{\chi} h^{2}$ from the value of the $\tilde{\tau}_{2}$ mass.

Higgs sectors must all be measured or constrained. The goals of the analysis must therefore be similar to that described here and if accessible use will be made of the decay chains considered above.

Although it is impossible to outline the required analyses for all possible models a few general observations may be made. Models in which stau co-annihilation dominates display similar phenomenology to the light slepton annihilation region, however the small mass difference between the $\tilde{\tau}_{1}$ and $\tilde{\chi}_{1}^{0}$ makes observation and measurement of the $\tilde{q}_{L} \rightarrow \tilde{\chi}_{2}^{0} q \rightarrow \tilde{\tau}_{1}^{ \pm} \tau^{\mp} q \rightarrow \tau^{ \pm} \tau^{\mp} q$ decay chain more difficult. In regions in which the $\tilde{\chi}_{1}^{0}$ possesses a significant Higgsino or Wino component (see e.g. [37, 38), leading to dominant (co-)annihilation to EW bosons the analysis would be qualitatively different to that described here and highly model dependent. If heavy Higgs annihilation is important techniques such as those described above for measuring its mass will be vital. When stop co-annihilation is enhanced the small mass difference between the strongly interacting $\tilde{t}_{1}$ and $\tilde{\chi}_{1}^{0}$ will likely make measurement of the masses very difficult indeed.

\section{Conclusions}

We have explored the potential of the LHC experiments for predicting the cosmological relic density of the LSP from detailed measurements of the SUSY spectrum and decay modes. No unification condition is imposed on the sparticle spectrum. We have focused on a model with essentially bino LSP and light sleptons, for which a detailed experimental study exists in the 
literature. We have examined the relative roles of the different measurement uncertainties, and studied the uncertainties due to SUSY parameters which are relevant for the relic density calculation, and are poorly, if at all, constrained by the measurements at the LHC. For the experimentally accessible measurements, we have highlighted the role of the measurement of the $\tau \tau$ edge. For the influence of badly measured parameters, the key issue is the observability of the heavy Higgses at the LHC. We have discussed in particular the prospect for observing the H/A in the cascade decays of the sparticles or in a SUSY decay mode. In case the position of the $\tau \tau$ edge can be controlled at the level of $\sim 1 \mathrm{GeV}$, and the LHC experiments can demonstrate that the Heavy Higgses $H, A$ have a mass in excess of $300 \mathrm{GeV}$, the value of the neutralino relic density can be predicted as:

$$
\Omega_{\chi} h^{2}=0.108 \pm 0.01(s t a t+s y s)_{-0.002}^{+0.00}(M(A))_{-0.011}^{+0.001}(\tan \beta)_{-0.005}^{+0.002}\left(m\left(\tilde{\tau}_{2}\right)\right)
$$

after three years of data taking at high luminosity, corresponding to an integrated luminosity of $300 \mathrm{fb}^{-1}$. In case no experimental information on the heavy Higgs can be extracted from the LHC data, it will only be possible to put an upper limit of approximately 0.12 on the neutralino relic density. The discovery of the decay of the heavy Higgses to SUSY particles is however most probably statistics limited, and a luminosity upgrade of the LHC might allow the discovery of this decay mode, thus yielding also a constraint on $\tan \beta$.

\section{Acknowledgments}

We would like to thank Genevieve Belanger for useful discussions on the usage of micrOMEGAs.

\section{References}

[1] C. L. Bennett et al., Astrophys. J. Suppl. 148 (2003) 1 arXiv:astro-ph/0302207.

[2] D. N. Spergel et al., Astrophys. J. Suppl. 148 (2003) 175 arXiv:astro-ph/0302209.

[3] H. V. Peiris et al., Astrophys. J. Suppl. 148 (2003) 213 arXiv:astro-ph/0302225.

[4] G. Polesello and D. R. Tovey, JHEP 0405 (2004) 071 arXiv:hep-ph/0403047.

[5] L. Alvarez-Gaume, J. Polchinski and M.B. Wise, Nucl. Phys. B221 (1983) 495; L. Ibanez, Phys. Lett. B118 (1982) 73; J. Ellis, D.V. Nanopoulos and K. Tamvakis, Phys. Lett. B121 (1983) 123; K. Inoue et al., Prog. Theor. Phys. 68 (1982) 927; A.H. Chamseddine, R. Arnowitt and P. Nath, Phys. Rev. Lett. 49 (1982) 970.

[6] M. Battaglia and M. E. Peskin, arXiv:hep-ph/0509135

[7] A. Birkedal et al., arXiv:hep-ph/0507214.

[8] J. A. Aguilar-Saavedra et al., arXiv:hep-ph/0511344.

[9] B. C. Allanach et al., in Proc. of the APS/DPF/DPB Summer Study on the Future of Particle Physics (Snowmass 2001) ed. N. Graf, Eur. Phys. J. C 25 (2002) 113 [eConf C010630 (2001) P125] arXiv:hep-ph/0202233. 
[10] M. Battaglia, A. De Roeck, J. R. Ellis, F. Gianotti, K. A. Olive and L. Pape, Eur. Phys. J. C 33, 273 (2004) arXiv:hep-ph/0306219.

[11] H. Baer, F. E. Paige, S. D. Protopescu and X. Tata, arXiv:hep-ph/0312045

[12] G. Belanger, F. Boudjema, A. Pukhov and A. Semenov, Comput. Phys. Commun. 149 (2002) 103 arXiv:hep-ph/0112278.

[13] H. Bachacou, I. Hinchliffe and F. E. Paige, Phys. Rev. D 62, 015009 (2000) hep-ph/9907518.

[14] ATLAS Collaboration, ATLAS detector and physics performance Technical Design Report, CERN/LHCC 99-14/15 (1999).

[15] B. C. Allanach, C. G. Lester, M. A. Parker and B. R. Webber, JHEP0009, 004 (2000) arXiv:hep-ph/0007009.

[16] B.K. Gjelsten, E. Lytken, D.J. Miller, P. Osland and G. Polesello, ATLAS internal note ATL-PHYS-2004-007 (2004), published in G. Weiglein et al. [LHC/LC Study Group], arXiv:hep-ph/0410364.

[17] B. K. Gjelsten, D. J. Miller and P. Osland, JHEP 0412 (2004) 003 arXiv:hep-ph/0410303.

[18] M. Hohlfeld, On the determination of Higgs parameters in the ATLAS experiment at the LHC, ATLAS internal note ATL-PHYS-2001-004 (2001).

[19] C. G. Lester, M. A. Parker and M. J. White, arXiv:hep-ph/0508143.

[20] B. K. Gjelsten, D. J. Miller and P. Osland, arXiv:hep-ph/0507232.

[21] T. Goto, K. Kawagoe and M. M. Nojiri, Phys. Rev. D 70 (2004) 075016 [Erratum-ibid. D 71 (2005) 059902] arXiv:hep-ph/0406317.

[22] A. J. Barr, Phys. Lett. B 596, 205 (2004) arXiv:hep-ph/0405052.

[23] J. A. Casas, A. Lleyda and C. Munoz, Nucl. Phys. B 471, 3 (1996) arXiv:hep-ph/9507294.

[24] A. Kusenko, P. Langacker and G. Segre, Phys. Rev. D 54, 5824 (1996) arXiv:hep-ph/9602414.

[25] A. Datta, A. Djouadi, M. Guchait and F. Moortgat, Nucl. Phys. B 681 (2004) 31 arXiv:hep-ph/0303095.

[26] G. Corcella et al., JHEP 0101, 010 (2001) arXiv:hep-ph/0011363; S. Moretti, K. Odagiri, P. Richardson, M. H. Seymour and B. R. Webber, JHEP 0204, 028 (2002) arXiv:hep-ph/0204123.

[27] W. Beenakker, R. Hopker, M. Spira and P. M. Zerwas, Nucl. Phys. B 492, 51 (1997) arXiv:hep-ph/9610490; M. Spira, arXiv:hep-ph/0211145. 
[28] M. Bisset, F. Moortgat and S. Moretti, Eur. Phys. J. C 30 (2003) 419 arXiv:hep-ph/0303093.

[29] F. Moortgat, S. Abdullin and D. Denegri, arXiv:hep-ph/0112046.

[30] C. Hansen, N. Gollub, K. Assamagan and T. Ekelof, arXiv:hep-ph/0504216.

[31] M. Spira, A. Djouadi, D. Graudenz and P. M. Zerwas, Nucl. Phys. B 453, 17 (1995) arXiv:hep-ph/9504378.

[32] S. Dittmaier, M. Kramer and M. Spira, Phys. Rev. D 70, 074010 (2004) arXiv:hep-ph/0309204.

[33] M. M. Nojiri, G. Polesello and D. R. Tovey, arXiv:hep-ph/0312317.

[34] B. C. Allanach, G. Belanger, F. Boudjema and A. Pukhov, JHEP 0412 (2004) 020 arXiv:hep-ph/0410091.

[35] J. Hisano, K. Kawagoe and M. M. Nojiri, Phys. Rev. D 68 (2003) 035007 arXiv:hep-ph/0304214.

[36] G. Polesello, J. Phys. G 30 (2004) 1185.

[37] H. Baer, A. Mustafayev, E. K. Park and S. Profumo, JHEP 0507 (2005) 046 arXiv:hep-ph/0505227.

[38] H. Baer, T. Krupovnickas, A. Mustafayev, E. K. Park, S. Profumo and X. Tata, arXiv:hep-ph/0511034. 